\title{
Perturbation-facilitated detection of the first quintet-quintet band in $\mathbf{C}_{\mathbf{2}}$
}

\author{
P. Bornhauser, ${ }^{1}$ R. Marquardt, ${ }^{2}$ C. Gourlaouen, ${ }^{2}$ G. Knopp,${ }^{1}$ M. Beck, ${ }^{1}$ T. Gerber,${ }^{1}$ \\ J. A. van Bokhoven, ${ }^{1,3}$ and P. P. Radi ${ }^{1, a)}$ \\ ${ }^{1}$ Paul Scherrer Institute, $\mathrm{CH}-5232$ Villigen, Switzerland \\ ${ }^{2}$ Laboratoire de Chimie Quantique, Institut de Chimie, Université de Strasbourg. 4, rue Blaise Pascal - \\ CS90032 67081 STRASBOURG CEDEX, France \\ ${ }^{3}$ Institute for Chemical and Bioengineering, ETHZ, Zürich, Switzerland
}

(Received 26 January 2015; accepted 19 February 2015; published online 6 March 2015)

\begin{abstract}
The first high-spin transition in $\mathrm{C}_{2}\left(1{ }^{5} \Pi_{\mathrm{u}}-1{ }^{5} \Pi_{\mathrm{g}}\right)$ is observed by perturbation-facilitated opticaloptical double resonance spectroscopy. The experiment is performed by applying unfolded two-color resonant four-wave mixing. $\mathrm{C}_{2}$ radicals in the initial a ${ }^{3} \Pi_{\mathrm{u}}, v=5$ state are produced by using a discharge source in a molecular beam environment. The final quintet state is excited via intermediate "gateway" states exhibiting both substantial triplet and quintet character due to a perturbation between the $1{ }^{5} \Pi_{\mathrm{g}}, v=0$ and the $\mathrm{d}^{3} \Pi_{\mathrm{g}}, v=6$ states. Fifty seven rotational transitions in the $\mathrm{P}, \mathrm{Q}$, and $\mathrm{R}$ branches of all spin sub-states are measured and yield accurate molecular constants of the newly found upper level $1{ }^{5} \Pi_{\mathrm{u}}$. In addition, satellite transitions $(\Delta J \neq \Delta N)$ are observed and allow an accurate determination of the spin-orbit constant. The results are compared with high-level $a b$ initio computations at the multi-reference configuration interaction level of theory. The high-lying quintet state is found to be predissociative and displays a shallow potential that accommodates three vibrational levels only. @ 2015 AIP Publishing LLC. [http://dx.doi.org/10.1063/1.4913925]
\end{abstract}

\section{INTRODUCTION}

Since the pioneering work of Wollaston ${ }^{1}$ and Swan, ${ }^{2}$ numerous electronic states of $\mathrm{C}_{2}$ have been investigated spectroscopically. Taking into account the dipole selection rule on spin, $\Delta S=0$ and considering the thermally populated singlet ground state $\left(\mathrm{X}^{1} \Sigma_{\mathrm{g}}^{+}\right)$and the low-lying triplet $\left(\mathrm{a}^{3} \Pi_{\mathrm{u}}\right)$ electronic state at only $\approx 720 \mathrm{~cm}^{-1}$ above, most studies involved high-lying singlet and triplet electronic states. A review of the research up to 1992 can be found in Ref. 3 . More recent work reported on an additional new band system $\left(\mathrm{d}^{3} \Pi_{\mathrm{g}}-\mathrm{c}^{3} \Sigma_{\mathrm{u}}^{+}\right)$observed by laser-induced fluorescence of excited radicals produced in an acetylene discharge. The first direct observation of the metastable $\mathrm{c}^{3} \Sigma_{\mathrm{u}}^{+}$allowed the determination of rotational constants and the vibrational origin. $^{4}$

Distinct from singlet and triplet electronic states, some of us unveiled the first high-spin state $\left(1^{5} \Pi_{\mathrm{g}}\right)$ of $\mathrm{C}_{2}$ in the course of a deperturbation study of the $\mathrm{d}^{3} \Pi_{\mathrm{g}}, v=6$ state. ${ }^{5}$ The detailed investigation of the spin-orbit interaction of the two electronic states unraveled major features of the so-called high-pressure bands of $\mathrm{C}_{2}$ initially observed a century ago. ${ }^{6}$ The non-thermal emission observed in numerous experimental environments is rationalized by taking into account perturbations yielding "gateway" states, i.e., rotational levels of the $\mathrm{d}^{3} \Pi_{\mathrm{g}}, v=6$ state exhibiting significant $1{ }^{5} \Pi_{\mathrm{g}}$ character through which all population flows from one electronic state to the other.

\footnotetext{
${ }^{a}$ Author to whom correspondence should be addressed. Electronic mail: peter.radi@psi.ch
}

Soon after the experimental characterization of the $1^{5} \Pi_{\mathrm{g}}$ state, $a b$ initio computations at the multi-reference configuration interaction level of theory of this state ${ }^{7}$ gave improved results compared to previous calculations of the quintet state by Kirby and Liu. ${ }^{8}$ The identity of the observed dark state has been confirmed as the $v=0$ level of the $1^{5} \Pi_{\mathrm{g}}$ electronic state. It was found that the wavefunctions for the $\mathrm{d}^{3} \Pi_{\mathrm{g}}, v=6$ level and the $1{ }^{5} \Pi_{\mathrm{g}}, v=0$ level overlap strongly, adding further evidence that $\mathrm{C}_{2}$ formed as a quintet can pass through "gateway" states in the $1{ }^{5} \Pi_{\mathrm{g}}, v=0$ level to emerge as $\mathrm{d}^{3} \Pi_{\mathrm{g}}, v=6$ level band fluorescence. It is important to mention that the equilibrium separation of the $1^{5} \Pi_{\mathrm{g}}$ is the largest among all states that correlate to ground state product carbon atoms, $\mathrm{C}\left({ }^{3} \mathrm{P}\right)$ (vide infra). The outer turning point of the potential curve is also the largest. Therefore, formation of $\mathrm{C}_{2}$ through collision of free carbon will lead to significant population of the metastable $1^{5} \Pi_{\mathrm{g}}$ state. ${ }^{9}$

Following closely our approach for the deperturbation of the $\mathrm{d}^{3} \Pi_{\mathrm{g}}, v=4$ state, ${ }^{10}$ the study revealing the $1^{5} \Pi_{\mathrm{g}}$ state has been performed by applying two-color resonant four-wave mixing (TC-RFWM). ${ }^{5}$ The sensitivity and selectivity of the method allowed us to observe and unambiguously assign a large number of perturbed transitions in the $(6,5)$ vibronic band of the Swan system $\left(\mathrm{d}^{3} \Pi_{\mathrm{g}}-\mathrm{a}^{3} \Pi_{\mathrm{u}}\right)$. In addition, many low-intensity excitations to "dark states" that are made visible by the electronic state mixing have been measured. These weak transitions appear because states in the vicinity of a perturbation are actually mixed eigenstates containing state character of both levels. A global fit of the line positions, to a conventional Hamiltonian for a linear diatomic molecule, has resulted in accurate molecular constants for both, the triplet and the quintet states for the first time. In addition, parameters 
for the spin-orbit and $L$-uncoupling interaction between the electronic states have been determined. Thus, the perturbation has been characterized in detail and is exactly calculable.

The mixing coefficients due to the perturbations are of particular interest for this work because the quintettriplet mixed levels can act as "gateways" or "windows" into the quintet manifold. The coefficients are quantified by considering that the spin-sublevels of the $d^{3} \Pi_{g}$ state are actually admixtures and not pure $\Omega$ states. In fact, the spin-orbit interaction $(A \hat{L} \cdot \hat{S})$ [see Eq. 1 in Ref. 5] produces eigenstates exhibiting significant mixtures of all three nominal spin-states, i.e., $\mathrm{d}^{3} \Pi_{2 \mathrm{~g}}, \mathrm{~d}^{3} \Pi_{1 \mathrm{~g}}$, and $\mathrm{d}^{3} \Pi_{0 \mathrm{~g}}$. Upon perturbation, the $1^{5} \Pi_{\mathrm{g}}$ spin substates, i.e., $1^{5} \Pi_{-1 \mathrm{~g}}, 1^{5} \Pi_{0 \mathrm{~g}}$, $1^{5} \Pi_{1 \mathrm{~g}}, 1{ }^{5} \Pi_{2 \mathrm{~g}}, 1{ }^{5} \Pi_{3 \mathrm{~g}}$, reduce their $1^{5} \Pi_{\mathrm{g}}$ character and gain $\mathrm{d}^{3} \Pi_{\mathrm{g}}$ character. Table I lists the fractional $\mathrm{d}^{3} \Pi_{\mathrm{g}}$ character gain of $1{ }^{5} \Pi_{\mathrm{g}}$ rotational terms for the five $\mathrm{F}$ components. Note that the spin-orbit interaction term in the Hamiltonian generates three manifolds in the $\mathrm{d}^{3} \Pi_{\mathrm{g}}$ state, i.e., $\mathrm{F}_{1}, \mathrm{~F}_{2}$, and $\mathrm{F}_{3}$ ordered by increasing energy. Since in this state the spinorbit constant $A$ is negative, the energies of the spin-substates are $E\left(\mathrm{~d}^{3} \Pi_{0 \mathrm{~g}}\left(\mathrm{~F}_{3}\right)\right)>E\left(\mathrm{~d}^{3} \Pi_{1 \mathrm{~g}}\left(\mathrm{~F}_{2}\right)\right)>E\left(\mathrm{~d}^{3} \Pi_{2 \mathrm{~g}}\left(\mathrm{~F}_{1}\right)\right)$. In contrast, the $1^{5} \Pi_{\mathrm{g}}$ state exhibits a positive $A$ constant, ${ }^{5}$ therefore the energies of the five quintet manifolds increase with increasing $\Omega$, i.e. $E\left(1^{5} \Pi_{-1 \mathrm{~g}}\left(\mathrm{~F}_{1}\right)\right)<E\left(1^{5} \Pi_{0 \mathrm{~g}}\left(\mathrm{~F}_{2}\right)\right)<E\left(1^{5} \Pi_{1 \mathrm{~g}}\left(\mathrm{~F}_{3}\right)\right)$ $<E\left(1^{5} \Pi_{2 \mathrm{~g}}\left(\mathrm{~F}_{4}\right)\right)<E\left(1^{5} \Pi_{3 \mathrm{~g}}\left(\mathrm{~F}_{5}\right)\right)$.

Fig. 1 shows how the large transition strength of the Swan system d ${ }^{3} \Pi_{g}-a{ }^{3} \Pi_{u}$ which is loaned to the quintet manifold is used to observe nominally forbidden transitions from the ${ }^{3} \Pi_{u}$ to the quintet manifold. The PUMP photons $\left(\lambda_{1}\right)$ excite from the initially populated $\mathrm{a}^{3} \Pi_{\mathrm{u}}, v=5$ level mixed "gateway" levels, i.e., nominal quintet states exhibiting significant triplet $\mathrm{d}^{3} \Pi_{\mathrm{g}}$ character through the perturbation (Table I). By applying a further excitation step $\left(\lambda_{2}\right)$, the dicarbon quintet manifold can be studied by using a specific optical-optical double-resonance (OODR) method termed "perturbation-facilitated optical-

TABLE I. Fractional triplet character of the rotational levels of the $1^{5} \Pi_{\mathrm{g}}$, $v=0$ vibronic level. Even and odd $J$ levels are of e and f symmetry, respectively.

\begin{tabular}{lccccc}
\hline \hline $\boldsymbol{J}$ & $\mathrm{F}_{1}$ & $\mathrm{~F}_{2}$ & $\mathrm{~F}_{3}$ & $\mathrm{~F}_{4}$ & $\mathrm{~F}_{5}$ \\
\hline 0 & & 0.04 & & & \\
1 & 0.00 & 0.12 & 0.32 & & \\
2 & 0.01 & 0.10 & 0.31 & 0.18 & \\
3 & 0.01 & 0.14 & 0.08 & 0.08 & 0.16 \\
4 & 0.01 & 0.34 & 0.07 & 0.05 & 0.17 \\
5 & 0.01 & 0.26 & 0.04 & 0.19 & 0.11 \\
6 & 0.01 & 0.09 & 0.03 & 0.06 & 0.09 \\
7 & 0.01 & 0.03 & 0.01 & 0.03 & 0.06 \\
8 & 0.01 & 0.02 & 0.01 & 0.02 & 0.06 \\
9 & 0.00 & 0.01 & 0.01 & 0.02 & 0.09 \\
10 & 0.00 & 0.00 & 0.00 & 0.02 & 0.24 \\
11 & 0.00 & 0.00 & 0.00 & 0.04 & 0.13 \\
12 & 0.00 & 0.00 & 0.00 & 0.47 & 0.03 \\
13 & 0.00 & 0.00 & 0.00 & 0.03 & 0.01 \\
14 & 0.00 & 0.00 & 0.00 & 0.01 & 0.01 \\
15 & 0.00 & 0.00 & 0.00 & 0.00 & 0.00 \\
16 & 0.00 & 0.00 & 0.00 & 0.00 & 0.00 \\
17 & 0.00 & 0.00 & 0.00 & 0.00 & 0.01 \\
\hline \hline
\end{tabular}

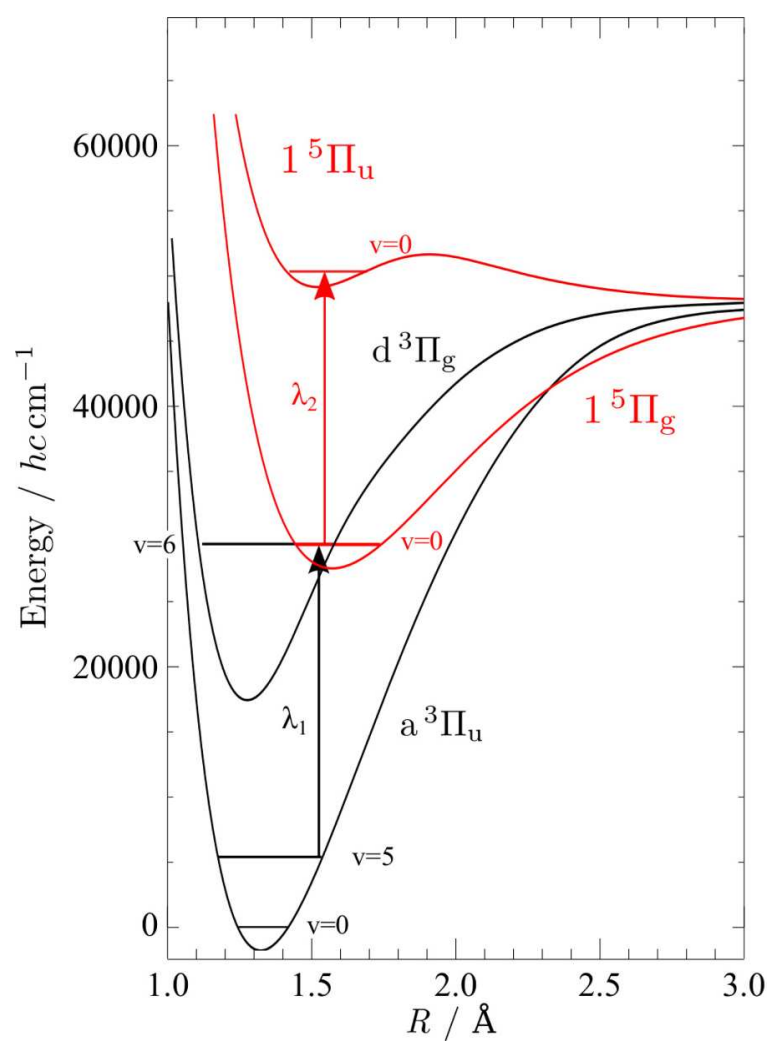

FIG. 1. PFOODR scheme for the detection of $1{ }^{5} \Pi_{u}$ in a potential energy diagram. The PUMP beams $\left(\lambda_{1}\right)$ excite to a mixed state (involving $1^{5} \Pi_{\mathrm{g}}$, $v=0)$ that gains triplet intensity from the bright Swan system $\left(\mathrm{d}^{3} \Pi_{\mathrm{g}}-\mathrm{a}^{3} \Pi_{\mathrm{u}}\right)$. From there, the PROBE beam $\left(\lambda_{2}\right)$ excites the $1{ }^{5} \Pi_{\mathrm{u}}, v=0$ state (red arrow). $A b$ initio potential energy curves are computed at the MRCI level of theory with a large basis set (see text for details).

optical double resonance" (PFOODR). PFOODR has been utilized to access dark triplet spin states from singlet ground states via singlet-triplet mixed levels as intermediate window states in two-step excitations. Mainly alkali metal dimers were studied by applying the technique (see Refs. 11-13). Investigations have been performed by using methods such as fluorescence excitation, ${ }^{14}$ dispersed fluorescence (DF) detection, ${ }^{13,15}$ or continuous-wave optical triple resonance spectroscopy. ${ }^{16}$ Recently, a perturbation-assisted approach to prepare population in high vibrational states of $\mathrm{CO}\left(\mathrm{X}^{1} \Sigma^{+}\right)$ was demonstrated. ${ }^{17}$ PFOODR spectroscopy by applying nonlinear methods has been demonstrated for the first time by some of us on $\mathrm{C}_{3},{ }^{18}$ where the (dark) triplet manifold of the tricarbon molecule has been reached from the singlet $\tilde{X}^{1} \Sigma_{\mathrm{g}}^{+}$ ground state by a two-step excitation via singlet-triplet mixed "gateway" levels uncovering the existence of a new triplet electronic state of presumably ${ }^{3} \Delta_{\mathrm{u}}$ symmetry.

The present work reports on the analysis of the first quintet-quintet transition of $\mathrm{C}_{2}$. In Sec. II, we summarize briefly the experimental environment established for PFOODR measurements. Section III reports on the general implementation of double-resonant four-wave mixing (TC-RFWM) (Sec. III A). It follows the listing of the measured transition frequencies of the $1{ }^{5} \Pi_{u}-1{ }^{5} \Pi_{\mathrm{g}}$ system obtained by applying the technique. The data reduction by performing a non-linear least squares fit to a conventional Hamiltonian is then outlined and yields molecular constants of the $1^{5} \Pi_{u}$ state (Sec. III B). 
In Sec. III C, we summarize briefly the ab initio computations of the excited states of $\mathrm{C}_{2}$ performed at the multi-reference configuration interaction (MRCI) level of theory that helped us rationalize the experimental data and obtain Fig. 1 and finalize the paper with Sec. IV.

\section{EXPERIMENT}

The experiments are performed in a molecular beam apparatus designed for simultaneous linear and non-linear spectroscopic measurements of stable and transient molecules. ${ }^{19} \mathrm{C}_{2}$ in the low-lying a ${ }^{3} \Pi_{\mathrm{u}}$ state was prepared in the molecular beam environment by expansion of $\approx 1 \%$ acetylene diluted in Ar buffer gas through a solenoid valve (General Valve, Parker). ${ }^{20}$ Typical backing pressures are between 5 and 11 bar. The molecular pulse has a duration of about $750 \mu \mathrm{s}$. Prior to the expansion, the pulse passes through a multichannel flow-body producing a two-dimensional flow which is pertinent to the slit geometry of the discharge source. ${ }^{21}$ The slit-expansion geometry increases the interaction volume between the crossing area of four-wave mixing laser beams and the molecular jet. Compared to a more common pinhole expansion, the sensitivity of the method can be enhanced by nearly two orders of magnitude due to the quadratic dependence of the four-wave mixing signal on interaction length. ${ }^{22}$ A pulsed applied potential difference of -800 to $-900 \mathrm{~V}$ and $\approx 2 \mu$ s duration is applied to the electrodes prior to the expansion zone. Trigger time-delays between the discharge and the molecular beam pulse were optimized to the maximum production of $\mathrm{C}_{2}$ molecules which were probed by four-wave mixing $\approx 5 \mathrm{~mm}$ downstream of the expansion region.

The optical setup has been described recently. ${ }^{18}$ Briefly, TC-RFWM is established by applying two separately pumped dye lasers (narrowscan, Radiant Dyes). The specified bandwidth of both laser systems is $\approx 0.04 \mathrm{~cm}^{-1}$. To obtain optimized spatial intensity profiles, both beams pass through spatial filters. Absolute wavelength calibration is performed for each scan step by a wavemeter (High Finess/Ångström, WS6). A forward BOXCARS configuration is set up by a combination of optical components as detailed in Ref. 23 and outlined briefly in the following. A $1000 \mathrm{~mm}$ focal length achromatic objective focuses three parallel propagating laser beams to pass along the three main diagonals of a parallelepiped and cross with a small angle of $\approx 1^{\circ}$. These beams are arranged orthogonally to the propagation direction of the molecular beam, hence, eliminating the Doppler broadening. The wave vectors, $\vec{k}_{i}$, of the interacting beams fulfill the phase-matching condition

$$
\overrightarrow{k_{1}}+\overrightarrow{k_{3}}=\overrightarrow{k_{2}}+\overrightarrow{k_{4}}
$$

and generate the signal beam $\vec{k}_{4}$ along the fourth (dark) diagonal. The two laser beams of equal frequencies are usually referred to as PUMP beams $\vec{k}_{1}, \vec{k}_{3}$, and the third beam, $\overrightarrow{k_{2}}$, as the PROBE beam. The SIGNAL beam is collimated by an achromatic objective $(\mathrm{FL}=1000 \mathrm{~mm})$. A number of spatial and spectral filters remove scattered light and unwanted fluorescence along the $\approx 2 \mathrm{~m}$ propagation of the signal in free-space. Further reduction of the stray light is achieved by a $30 \mathrm{~mm}$ focal lens and a $50 \mu \mathrm{m}$ pinhole in front of a photomultiplier tube. Typically $20-30$ pulses are averaged per scan step $\left(5 \times 10^{-4} \mathrm{~nm} / \mathrm{step}\right)$ of the dye-laser on the oscilloscope and transferred to a personal computer (PC) for further analysis.

\section{RESULTS AND DISCUSSION}

\section{A. Perturbation facilitated TC-RFWM}

The general implementation of perturbation-facilitated TC-RFWM to characterize the first quintet-quintet transition of $\mathrm{C}_{2}$ is depicted in Fig. 2 and examples are shown in Fig. 3. In panel (a) of Fig. 3, the intermediate rotational state $|i\rangle=\left|\Psi_{1}\right\rangle$ is the $\mathrm{F}_{5}(4)$ level of the $1^{5} \Pi_{\mathrm{g}}, v=0$ state. As mentioned in the Introduction, the spin-sublevels are not pure $\Omega$ states. Upon perturbation, the $\mathrm{F}_{5}(4)$ level gains $17 \%$ triplet character (Table I), i.e., $d^{3} \Pi_{2 g}(0.3 \%), d^{3} \Pi_{1 g}(0.9 \%)$, and d ${ }^{3} \Pi_{0 \mathrm{~g}}(15.8 \%)$ and its quintet character is reduced to $83 \%$, i.e., $1^{5} \Pi_{-1 g}$ $(0.2 \%), 1^{5} \Pi_{0 \mathrm{~g}}(3.4 \%), 1^{5} \Pi_{1 \mathrm{~g}}(25.3 \%), 1^{5} \Pi_{2 \mathrm{~g}}(13.3 \%), 1^{5} \Pi_{3 \mathrm{~g}}$ $(40.9 \%)$.

The $\mathrm{F}_{5}(4)$ state is excited from the thermally populated $\mathrm{F}_{3}(3)$ of the a ${ }^{3} \Pi_{\mathrm{u}}, v=5$ level by tuning the PUMP beams to the ${ }^{\mathrm{s}} \mathrm{R}_{53}(3)$ transition of the $(0,5)$ band of the $1^{5} \Pi_{\mathrm{g}}-\mathrm{a}{ }^{3} \Pi_{\mathrm{u}}$ electronic system at $21411.09 \mathrm{~cm}^{-1}$. The perturbation shifts this line by $-0.58 \mathrm{~cm}^{-1}$ from its unperturbed position. The latter is obtained by a simulation model omitting perturbation effects as detailed in Ref. 5. A relatively simple PFOODR spectrum (Fig. 3(a)) is obtained by scanning the PROBE near $21800 \mathrm{~cm}^{-1}$. Assuming either Hund's case (a) or (b) for the upper and lower quintet states, $1^{5} \Pi_{\mathrm{u}}$ and $1^{5} \Pi_{\mathrm{g}}$, respectively, and disregarding $\Lambda$-type doubling, we expect five subbands for a ${ }^{5} \Pi_{\mathrm{u}}-{ }^{5} \Pi_{\mathrm{g}}$ transition, i.e., ${ }^{5} \Pi_{-1 \mathrm{~g}}-{ }^{5} \Pi_{-1 \mathrm{~g}}\left(\mathrm{~F}_{1}-\mathrm{F}_{1}\right)$,

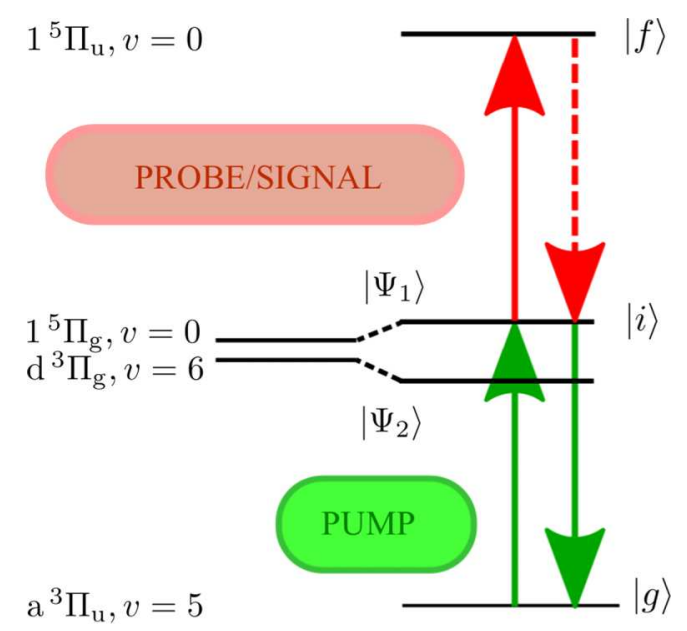

FIG. 2. PFOODR by applying TC-RFWM. The energy level diagram shows how the $1^{5} \Pi_{\mathrm{u}}$ level is linked to the $\mathrm{a}^{3} \Pi_{\mathrm{u}}, v=5$ state in the PFOODR experiment. The PUMP beams excite rotational levels in the thermally populated $\mathrm{a}^{3} \Pi_{\mathrm{u}}, v=5$ level to the nominal $1{ }^{5} \Pi_{\mathrm{g}}, v=0$ state. The spin-forbidden transition gains intensity from the spin-orbit interaction between the $1^{5} \Pi_{\mathrm{g}}, v=0$ and $\mathrm{d}^{3} \Pi_{\mathrm{g}}, v=6$ levels yielding a significant triplet character in the mixed state $\left|\Psi_{1}\right\rangle$. Subsequently, the PROBE beam links $\left|\Psi_{1}\right\rangle$ to the final rotational level in the $1^{5} \Pi_{\mathrm{u}}, v=0$ state. The SIGNAL beam exhibits the same frequency (broken line) and is emitted in a direction according to the phase matching condition (Eq. (1)). 


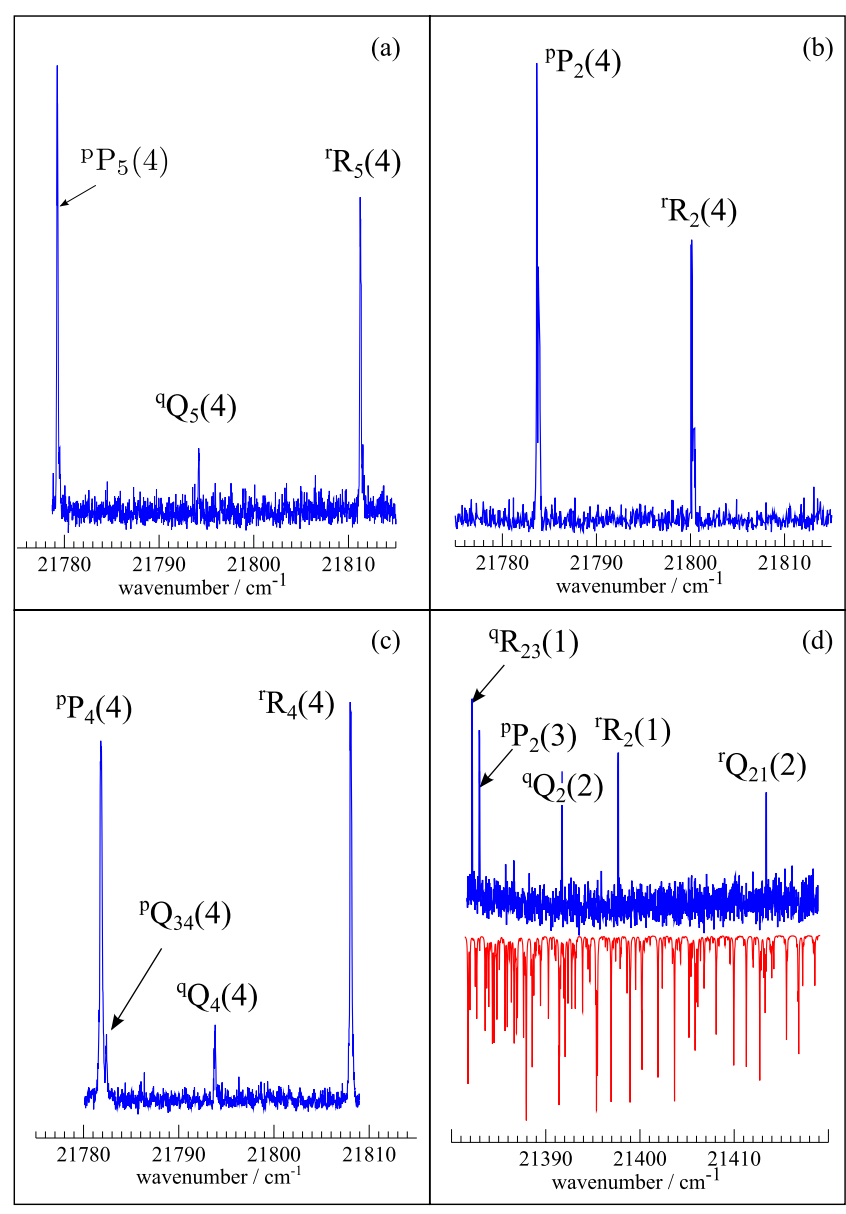

FIG. 3. PFOODR by TC-RFWM. Panels (a)-(c) are obtained by labeling selected intermediate levels $|i\rangle$ exhibiting significant $1{ }^{5} \Pi_{\mathrm{g}}$ character by the PUMP beams and scanning the PROBE beam (see Fig. 2). From the nominal $F_{5}(4)$ level of the "dark" $1{ }^{5} \Pi_{\mathrm{g}}$ electronic state with $83 \%$ quintet character, three transitions to $1^{5} \Pi_{\mathrm{u}}$ state in the $\mathrm{P}, \mathrm{Q}$, and $\mathrm{R}$ branches are observed (a). By addressing the $\mathrm{F}_{2}(4)$ intermediate level shown in panel (b), the $\mathrm{Q}$ branch is not observed because this spin-substate is mainly a $\Omega=0$ state $\left({ }^{5} \Pi_{0 \mathrm{~g}}\right)$. Panel (c) is obtained by tuning the PUMP laser beams to excite the intermediate level $\mathrm{F}_{4}(4)$. In addition to the three spin-allowed transitions in the $\mathrm{P}, \mathrm{Q}$, and $R$ branches, a weak satellite is observed linking the $F_{4}$ level with a $F_{3}$ term. Panel (d) shows exclusively rotational transitions from the $\mathrm{a}^{3} \Pi_{\mathrm{u}}, v=5$ state to levels of the "dark" $1{ }^{5} \Pi_{\mathrm{g}}$ state. Here, the PROBE laser is fixed on the ${ }^{\mathrm{q}} \mathrm{P}_{2}(2)$ transition of the $1^{5} \Pi_{\mathrm{u}}-1^{5} \Pi_{\mathrm{g}}$ system and the PUMP laser is scanned in the wavenumber region of the $\Delta v=1$ sequence bands of the Swan system. For comparison, a simulation of the dense and complex (one-photon) absorption spectrum is shown in the inverted trace.

${ }^{5} \Pi_{0 \mathrm{~g}}-{ }^{5} \Pi_{0 \mathrm{~g}}\left(\mathrm{~F}_{2}-\mathrm{F}_{2}\right),{ }^{5} \Pi_{1 \mathrm{~g}}-{ }^{5} \Pi_{1 \mathrm{~g}}\left(\mathrm{~F}_{3}-\mathrm{F}_{3}\right),{ }^{5} \Pi_{2 \mathrm{~g}}-{ }^{5} \Pi_{2 \mathrm{~g}}\left(\mathrm{~F}_{4}\right.$ $\left.-\mathrm{F}_{4}\right)$, and ${ }^{5} \Pi_{3 \mathrm{~g}}-{ }^{5} \Pi_{3 \mathrm{~g}}\left(\mathrm{~F}_{5}-\mathrm{F}_{5}\right)$. Each sub-band has a strong $\mathrm{P}$ and $\mathrm{R}$ line and with the exception of ${ }^{5} \Pi_{0 \mathrm{~g}}-{ }^{5} \Pi_{0 \mathrm{~g}}$ a weak $\mathrm{Q}$ line. Thus, the $\mathrm{P}, \mathrm{Q}$, and $\mathrm{R}$ branches for the $\mathrm{F}_{5}-\mathrm{F}_{5}$ transitions in Fig. 3(a) are assigned in a straightforward manner and yield the energies of the upper $\mathrm{F}_{5}(3), \mathrm{F}_{5}(4)$, and $\mathrm{F}_{5}(5)$ levels of the $1^{5} \Pi_{\mathrm{u}}$ state, respectively.

Exciting the heavily perturbed $\mathrm{F}_{2}(4)$ with $34 \%$ triplet character (Table I) by the $(0,5) 1^{5} \Pi_{g}-a^{3} \Pi_{u}{ }^{r} R_{21}(3)$ transition at $21419.77 \mathrm{~cm}^{-1}$ yields the PFOODR spectrum shown in the Fig. 3(b). Since $\Omega=0$, no Q branch is discernible in agreement with the expectation for the ${ }^{5} \Pi_{0 \mathrm{~g}}-{ }^{5} \Pi_{0 \mathrm{~g}}$ transition. Again, by considering the intermediate level labeling of $\mathrm{F}_{2}(4)$ the assignment of the upper rotational levels to $F_{2}(5)$ and $F_{2}(3)$ is straightforward.
A few satellite transitions $(\Delta J \neq \Delta N)$ are also observed. An example is shown in Fig. 3(c). The intermediate level $\mathrm{F}_{4}(4)$ is pumped by the ${ }^{\mathrm{r}} \mathrm{R}_{43}(3)$ transition in the $(0,5)$ band of the $1^{5} \Pi_{\mathrm{g}}-\mathrm{a}{ }^{3} \Pi_{\mathrm{u}}$ system at $21398.64 \mathrm{~cm}^{-1}$. Apart from the main $\mathrm{P}, \mathrm{Q}$, and $\mathrm{R}$ branches, a weak feature is observed that is assigned to ${ }^{\mathrm{P}} \mathrm{Q}_{34}(4)$. This branch would not be observed if the quintet states would strictly belong to Hund's case (b). However, towards low $J$ values, the states belong neither strictly to case (a) nor strictly to case (b) rendering the satellites visible. In total, 7 satellite transitions were observed, i.e., ${ }^{\mathrm{r}} \mathrm{Q}_{32}(2),{ }^{\mathrm{r}} \mathrm{R}_{43}(1),{ }^{\mathrm{p}} \mathrm{R}_{23}(2),{ }^{\mathrm{r}} \mathrm{Q}_{43}(2),{ }^{\mathrm{r}} \mathrm{R}_{43}(2),{ }^{\mathrm{p}} \mathrm{Q}_{23}(5)$, and ${ }^{\mathrm{P}} \mathrm{Q}_{34}(4)$. These cross transitions between different $\mathrm{F}$ levels allow the determination of accurate spin-orbit $A$ constants (vide infra).

The dominant quintet-quintet character of the $|f\rangle-|i\rangle$ transitions to the final state $1^{5} \Pi_{u}$ state (Fig. 2) is further evidenced by applying a "spin-filter" measurement. ${ }^{18}$ Panel (d) in Fig. 3 is obtained by tuning the PROBE laser to the ${ }^{\mathrm{q}} \mathrm{P}_{2}(2)$ transition of the $(0,0) 1^{5} \Pi_{\mathrm{u}}-1{ }^{5} \Pi_{\mathrm{g}}$ system at $21790.03 \mathrm{~cm}^{-1}$ (see Table II) and scanning the PUMP lasers in the wavenumber region of the $\Delta v=1$ sequence bands of the $\mathrm{d}^{3} \Pi_{\mathrm{g}}-\mathrm{a}{ }^{3} \Pi_{\mathrm{u}}$ transition around $21400 \mathrm{~cm}^{-1}$. A typical absorption spectrum of this region is simulated and shown with inverted intensities (red trace) in the figure. This region features a highly dense and complex spectrum of rotational transitions under the experimental conditions of the investigation $\left(T_{\mathrm{ROT}} \approx 70 \mathrm{~K}, T_{\mathrm{vib}} \approx 3000 \mathrm{~K}\right)$. In contrast, by applying TC-RFWM, only transitions to the intermediate level $\mathrm{F}_{2}(2)$ of the $1^{5} \Pi_{\mathrm{g}}$ electronic state are observed and rationalized by the double-resonance selection rules. This intermediate level still exhibits, upon perturbation, $90 \%$ quintet character. As a consequence, exclusively weak transitions from the a ${ }^{3} \Pi_{u}$ state to the "dark" level are discerned. These minor transitions to the perturbing states are generally very difficult to observe with more common spectroscopic methods such as absorption or laser-induced fluorescence.

\section{B. Rotational assignment and fitting}

Table II lists 57 rotational transitions of the $(0,0) 1^{5} \Pi_{u}$ $-1^{5} \Pi_{\mathrm{g}}$ system that are assigned (second column) in a straightforward manner by intermediate labeling. In particular, the upper term values (first column) of the $1^{5} \Pi_{u}, v=0$ state are measured by applying the unfolded scheme of TC-RFWM depicted in Fig. 2. The initial pump transitions (third column) to the gateway levels (fourth column) occur in the $(0,5)$ band of the $1^{5} \Pi_{\mathrm{g}}-\mathrm{a}{ }^{3} \Pi_{\mathrm{u}}$ electronic system. The gateway levels addressed exhibit dominantly quintet character facilitating transitions to the $1^{5} \Pi_{\mathrm{u}}$ state. Note that a selected gateway level can be excited by different initial transitions. For example, $\mathrm{F}_{1}(4)$ of the $1^{5} \Pi_{\mathrm{g}}, v=0$ state is populated by the $\mathrm{R}_{1}(3)$ and $\mathrm{P}_{1}(5)$ transitions from the a ${ }^{3} \Pi_{\mathrm{u}}, v=5$ state at 21403.42 and $21379.12 \mathrm{~cm}^{-1}$, respectively. The second transition to the ${ }^{5} \Pi_{u}, v=0$ to the $\mathrm{F}_{1}(3)$ or $\mathrm{F}_{1}(5)$ terms is then performed in the $\mathrm{R}$ or $\mathrm{P}$ branch of the quintet-quintet transition. It is important to mention that intensities of two-step excitations by applying TC-RFWM are dependent on geometric factors ${ }^{24}$ through which the polarization configuration of the PUMP and PROBE beam are taken into account. However, since 
TABLE II. PFOODR by TC-RFWM. The initial transition occurs between the $\mathrm{a}^{3} \Pi_{\mathrm{u}}, \mathrm{v}=5$ and the "dark" perturbing $1^{5} \Pi_{\mathrm{g}}, \mathrm{v}=0$ states. The final state is the excited $1^{5} \Pi_{\mathrm{u}}$ state (see text for details). Transitions and residuals are in $\mathrm{cm}^{-1}$.

\begin{tabular}{|c|c|c|c|c|c|}
\hline $\begin{array}{l}\text { Upper } \\
\text { level }\end{array}$ & Assignment & $\begin{array}{c}\text { Initial } \\
\text { transition }\end{array}$ & $\begin{array}{c}\text { Gateway } \\
\text { level }\end{array}$ & Observed & Residuals \\
\hline F1e(1) & qP1e(2) & qQ12(2) & Fle(2) & 21790.22 & 0.02 \\
\hline $\mathrm{F} 1 \mathrm{f}(2)$ & qQ1e(2) & qQ12(2) & $\mathrm{F} 1 \mathrm{e}(2)$ & 21793.33 & -0.01 \\
\hline F1e(3) & qR1e(2) & qQ12(2) & F1e(2) & 21798.02 & 0.02 \\
\hline $\mathrm{F} 1 \mathrm{f}(2)$ & qP1f(3) & P1(4) & F1f(3) & 21788.50 & -0.03 \\
\hline F1f(4) & $\operatorname{rR} 1 f(3)$ & P1(4) & $\mathrm{F} 1 \mathrm{f}(3)$ & 21799.85 & 0.01 \\
\hline F1e(3) & $\mathrm{pP} 1 \mathrm{e}(4)$ & $\mathrm{P} 1(5)$ & F1e(4) & 21787.08 & 0.01 \\
\hline F1e(5) & rR1e(4) & $\mathrm{P} 1(5)$ & F1e(4) & 21802.08 & 0.03 \\
\hline F1e(3) & $\mathrm{pP} 1 \mathrm{e}(4)$ & R1(3) & F1e(4) & 21787.06 & -0.01 \\
\hline F1e(5) & rR1e(4) & $\mathrm{R} 1(3)$ & F1e(4) & 21802.00 & -0.04 \\
\hline$F 2 f(0)$ & $\mathrm{qP} 2 \mathrm{f}(1)$ & $\mathrm{qP} 2(2)$ & $\mathrm{F} 2 \mathrm{f}(1)$ & 21791.96 & 0.00 \\
\hline$F 2 f(2)$ & $\mathrm{qR} 2 \mathrm{f}(1)$ & $\mathrm{qP} 2(2)$ & $\mathrm{F} 2 \mathrm{f}(1)$ & 21796.39 & 0.01 \\
\hline $\mathrm{F} 2 \mathrm{e}(1)$ & $\mathrm{qP} 2 \mathrm{e}(2)$ & $\mathrm{P} 2(3)$ & $\mathrm{F} 2 \mathrm{e}(2)$ & 21790.03 & -0.02 \\
\hline $\mathrm{F} 2 \mathrm{e}(3)$ & $\mathrm{qR} 2 \mathrm{e}(2)$ & $\mathrm{P} 2(3)$ & $\mathrm{F} 2 \mathrm{e}(2)$ & 21798.00 & 0.01 \\
\hline $\mathrm{F} 2 \mathrm{e}(1)$ & $\mathrm{qP} 2 \mathrm{e}(2)$ & rQ21(2) & $\mathrm{F} 2 \mathrm{e}(2)$ & 21790.03 & 0.00 \\
\hline $\mathrm{F} 2 \mathrm{e}(3)$ & qR2e(2) & rQ21(2) & $\mathrm{F} 2 \mathrm{e}(2)$ & 21797.98 & -0.01 \\
\hline $\mathrm{F} 3 \mathrm{f}(2)$ & rQ32e(2) & rQ21(2) & $\mathrm{F} 2 \mathrm{e}(2)$ & 21803.69 & 0.00 \\
\hline $\mathrm{F} 2 \mathrm{e}(3)$ & $\mathrm{pP} 2 \mathrm{e}(4)$ & P21(5) & $\mathrm{F} 2 \mathrm{e}(4)$ & 21783.65 & 0.00 \\
\hline $\mathrm{F} 2 \mathrm{e}(5)$ & $\mathrm{rR} 2 \mathrm{e}(4)$ & P21(5) & $\mathrm{F} 2 \mathrm{e}(4)$ & 21800.11 & 0.00 \\
\hline $\mathrm{F} 2 \mathrm{f}(4)$ & pP2f(5) & rR21(4) & $\mathrm{F} 2 \mathrm{f}(5)$ & 21785.50 & -0.01 \\
\hline$F 2 f(6)$ & rR2f(5) & rR21(4) & $\mathrm{F} 2 \mathrm{f}(5)$ & 21806.91 & -0.02 \\
\hline $\mathrm{F} 2 \mathrm{e}(5)$ & $\mathrm{pP} 2 \mathrm{e}(6)$ & $\mathrm{qR} 21(5)$ & $\mathrm{F} 2 \mathrm{e}(6)$ & 21782.63 & -0.01 \\
\hline $\mathrm{F} 2 \mathrm{e}(7)$ & rR2e(6) & $\mathrm{qR} 21(5)$ & $\mathrm{F} 2 \mathrm{e}(6)$ & 21809.06 & -0.01 \\
\hline $\mathrm{F} 3 \mathrm{e}(1)$ & qQ3f(1) & rP32(2) & F3f(1) & 21789.72 & -0.01 \\
\hline F3f(2) & qR3f(1) & rP32(2) & F3f(1) & 21792.37 & -0.01 \\
\hline $\mathrm{F} 4 \mathrm{f}(2)$ & rR43f(1) & rP32(2) & $\mathrm{F} 3 \mathrm{f}(1)$ & 21802.08 & 0.00 \\
\hline $\mathrm{F} 2 \mathrm{e}(3)$ & $\mathrm{pR} 23 \mathrm{e}(2)$ & qP32(3) & $\mathrm{F} 3 \mathrm{e}(2)$ & 21785.35 & 0.00 \\
\hline $\mathrm{F} 3 \mathrm{e}(1)$ & $\mathrm{qP} 3 \mathrm{e}(2)$ & $\mathrm{qP} 32(3)$ & $\mathrm{F} 3 \mathrm{e}(2)$ & 21788.40 & -0.01 \\
\hline F3f(2) & qQ3e(2) & qP32(3) & $\mathrm{F} 3 \mathrm{e}(2)$ & 21791.08 & 0.02 \\
\hline $\mathrm{F} 3 \mathrm{e}(3)$ & qR3e(2) & qP32(3) & $\mathrm{F} 3 \mathrm{e}(2)$ & 21795.22 & 0.00 \\
\hline $\mathrm{F} 4 \mathrm{f}(2)$ & $\mathrm{rQ43e}(2)$ & qP32(3) & $\mathrm{F} 3 \mathrm{e}(2)$ & 21800.77 & 0.01 \\
\hline $\mathrm{F} 4 \mathrm{e}(3)$ & $\mathrm{rR} 43 \mathrm{e}(2)$ & qP32(3) & $\mathrm{F} 3 \mathrm{e}(2)$ & 21803.18 & 0.01 \\
\hline $\mathrm{F} 3 \mathrm{e}(3)$ & $\mathrm{pP} 3 \mathrm{e}(4)$ & pP32(5) & $\mathrm{F} 3 \mathrm{e}(4)$ & 21784.11 & 0.01 \\
\hline $\mathrm{F} 3 \mathrm{e}(5)$ & rR3e(4) & pP32(5) & $\mathrm{F} 3 \mathrm{e}(4)$ & 21804.08 & 0.01 \\
\hline $\mathrm{F} 3 \mathrm{f}(4)$ & pP3f(5) & P32(6) & F3f(5) & 21781.94 & 0.00 \\
\hline $\mathrm{F} 2 \mathrm{e}(5)$ & pQ23f(5) & rR32(4) & F3f(5) & 21780.05 & 0.02 \\
\hline F3f(4) & pP3f(5) & rR32(4) & F3f(5) & 21781.93 & 0.00 \\
\hline $\mathrm{F} 3 \mathrm{e}(5)$ & qQ3f(5) & rR32(4) & F3f(5) & 21793.41 & 0.01 \\
\hline F3f(6) & rR3f(5) & rR32(4) & F3f(5) & 21807.60 & 0.01 \\
\hline $\mathrm{F} 4 \mathrm{e}(3)$ & $\mathrm{pP} 4 \mathrm{e}(4)$ & sR42(3) & $\mathrm{F} 4 \mathrm{e}(4)$ & 21781.09 & -0.03 \\
\hline F3f(4) & pQ34e(4) & sR42(3) & $\mathrm{F} 4 \mathrm{e}(4)$ & 21781.66 & -0.02 \\
\hline $\mathrm{F} 4 \mathrm{f}(4)$ & qQ4e(4) & sR42(3) & $\mathrm{F} 4 \mathrm{e}(4)$ & 21793.04 & -0.01 \\
\hline $\mathrm{F} 4 \mathrm{e}(5)$ & rR4e(4) & sR42(3) & $\mathrm{F} 4 \mathrm{e}(4)$ & 21807.32 & -0.03 \\
\hline $\mathrm{F} 4 \mathrm{f}(4)$ & $\mathrm{pP} 4 \mathrm{f}(5)$ & $\mathrm{qP} 42(6)$ & $\mathrm{F} 4 \mathrm{f}(5)$ & 21779.62 & 0.00 \\
\hline $\mathrm{F} 4 \mathrm{f}(6)$ & $\operatorname{rR4f(5)}$ & $\mathrm{qP} 42(6)$ & $\mathrm{F} 4 \mathrm{f}(5)$ & 21810.87 & 0.01 \\
\hline $\mathrm{F} 4 \mathrm{e}(5)$ & $\mathrm{pP} 4 \mathrm{e}(6)$ & $\mathrm{qP} 42(7)$ & $\mathrm{F} 4 \mathrm{e}(6)$ & 21778.36 & 0.02 \\
\hline $\mathrm{F} 4 \mathrm{e}(7)$ & $\mathrm{rR} 4 \mathrm{e}(6)$ & $\mathrm{qP} 42(7)$ & $\mathrm{F} 4 \mathrm{e}(6)$ & 21814.56 & 0.02 \\
\hline $\mathrm{F} 4 \mathrm{e}(11)$ & $\mathrm{pP} 4 \mathrm{e}(12)$ & $\mathrm{tR} 41(11)$ & $\mathrm{F} 4 \mathrm{e}(12)$ & 21771.94 & 0.00 \\
\hline $\mathrm{F} 4 \mathrm{e}(13)$ & $\mathrm{rR} 4 \mathrm{e}(12)$ & tR41(11) & $\mathrm{F} 4 \mathrm{e}(12)$ & 21837.47 & -0.01 \\
\hline F5e(3) & pP5e(4) & sR53(3) & F5e(4) & 21779.27 & 0.02 \\
\hline F5f(4) & qQ5e(4) & sR53(3) & $\mathrm{F} 5 \mathrm{e}(4)$ & 21794.20 & 0.04 \\
\hline F5e(5) & rR5e(4) & sR53(3) & $\mathrm{F} 5 \mathrm{e}(4)$ & 21811.27 & 0.02 \\
\hline F5f(8) & pP5f(9) & tR52(8) & F5f(9) & 21773.42 & -0.05 \\
\hline F5f $(10)$ & rR5f(9) & tR52(8) & F5f(9) & 21829.39 & 0.01 \\
\hline F5e(9) & pP5e(10) & tR52(9) & F5e(10) & 21772.40 & 0.00 \\
\hline F5e(11) & rR5e(10) & tR52(9) & F5e(10) & 21833.13 & -0.01 \\
\hline F5f $(10)$ & pP5f(11) & tR52(10) & F5f(11) & 21773.24 & 0.01 \\
\hline F5f(12) & $\operatorname{rR5f}(11)$ & tR52(10) & F5f(11) & 21838.84 & 0.02 \\
\hline
\end{tabular}

parallel polarizations are used for all input beams, only minor differences of the relative intensities of the PROBE resonances according to the nature of their rotational branch and the rotational branch of the PUMP transition are expected. For example, the spectrum obtained by PUMPing the $F_{1}(4)$ level of the $1^{5} \Pi_{\mathrm{g}}, v=0$ state by the $\mathrm{R}_{1}(3)$ and $\mathrm{P}_{1}(5)$ yields similar intensities for the ${ }^{\mathrm{P}} \mathrm{P}_{1}(4)$ and ${ }^{\mathrm{r}} \mathrm{R}_{1}(4) \mathrm{PROBE}$ transitions in the $1^{5} \Pi_{\mathrm{g}}-1{ }^{5} \Pi_{\mathrm{u}}$ system.

Table III lists the molecular constants that are obtained by performing a least-squares optimization procedure to the 57 transitions listed in Table II using pgopher. ${ }^{26}$ As detailed in our preceding publication, ${ }^{5}$ a conventional Hamiltonian for a linear molecule ${ }^{27}$ is applied. The matrix elements listed in Ref. 5 for the $1^{5} \Pi_{\mathrm{g}}$ state are slightly modified for the $e$ and $f$ sublevels of the $1{ }^{5} \Pi_{u}$ state, i.e.,

$$
\begin{aligned}
\left\langle{ }^{5} \Pi_{-1}|\mathrm{H}|^{5} \Pi_{-1}\right\rangle= & T+B(x+2) \\
& -D\left(x^{2}+8 x+4\right)-2 A+4 \lambda, \\
\left\langle{ }^{5} \Pi_{0}|\mathrm{H}|^{5} \Pi_{0}\right\rangle= & T+B(x+6) \\
& -D\left(x^{2}+22 x+36\right)-A-2 \lambda \pm 3 o, \\
\left\langle{ }^{5} \Pi_{1}|\mathrm{H}|^{5} \Pi_{1}\right\rangle= & T+B(x+6) \\
& -D\left(x^{2}+24 x+24\right)-4 \lambda, \\
\left\langle{ }^{5} \Pi_{2}|\mathrm{H}|^{5} \Pi_{2}\right\rangle= & T+B(x+2) \\
& -D\left(x^{2}+14 x-32\right)+A-2 \lambda, \\
\left\langle{ }^{5} \Pi_{3}|\mathrm{H}|^{5} \Pi_{3}\right\rangle= & T+B(x-6) \\
\left\langle{ }^{5} \Pi_{-1}|\mathrm{H}|^{5} \Pi_{0}\right\rangle= & -2 \sqrt{x}[B-2 D(x+4)], \\
\left\langle{ }^{5} \Pi_{-1}|\mathrm{H}|^{5} \Pi_{1}\right\rangle= & \sqrt{6}(2 D x \pm o), \\
\left\langle{ }^{5} \Pi_{0}|\mathrm{H}|^{5} \Pi_{1}\right\rangle= & -\sqrt{6 x}[B-2 D(x+6)], \\
\left\langle{ }^{5} \Pi_{0}|\mathrm{H}|{ }^{5} \Pi_{2}\right\rangle= & -6 D \sqrt{x(x-2)}, \\
\left\langle{ }^{5} \Pi_{1}|\mathrm{H}|^{5} \Pi_{2}\right\rangle= & -\sqrt{6(x-2)}[B-2 D(x+4)], \\
\left\langle{ }^{5} \Pi_{1}|\mathrm{H}|^{5} \Pi_{3}\right\rangle= & -2 D \sqrt{6(x-2)(x-6)}, \\
\left\langle{ }^{5} \Pi_{2}|\mathrm{H}|{ }^{5} \Pi_{3}\right\rangle= & -2 \sqrt{x-6}[B-2 D(x-2)], \\
= &
\end{aligned}
$$

where $x=J(J+1)$. The \pm signs before $o$ refer to the $f$ and $e$ sublevels, respectively.

All parameters of the $1^{5} \Pi_{u}$ state were fitted simultaneously until the best possible fit was reached. The molecular constants of the $1^{5} \Pi_{\mathrm{g}}$ state were fixed at their values determined in our previous report. ${ }^{5}$ An excellent agreement between the observed transitions and the simulation is obtained. The root mean square (rms) value for the data reduction is $0.024 \mathrm{~cm}^{-1}$ which is approximately half of the specified laser bandwidth of the dye lasers. Alternatively, we have performed a least-squares fit to the term energies of the $1^{5} \Pi_{\mathrm{u}}$ state by taking into account $1^{5} \Pi_{\mathrm{u}}-1{ }^{5} \Pi_{\mathrm{g}}$ transitions and the determined term energies of the $1^{5} \Pi_{\mathrm{g}}$ state. ${ }^{5}$ The results of this data reduction improved the rms value to $0.017 \mathrm{~cm}^{-1}$. Note that inclusion of the centrifugal distortion constant $D$ improved the rms value of the fit significantly from 0.028 to $0.017 \mathrm{~cm}^{-1}$. However, the obtained $D$ constant has to be taken cautiously, because only transitions exhibiting relatively low $J(\leq 13)$ quantum numbers have been measured. In fact, the optimized value of $10 \times 10^{-6} \mathrm{~cm}^{-1}$ appears to be 
TABLE III. Optimized molecular constants for the $1^{5} \Pi_{\mathrm{u}}$ state. For comparison, parameters for the $1{ }^{5} \Pi_{\mathrm{g}}, \mathrm{d}{ }^{3} \Pi_{\mathrm{g}}$, and $\mathrm{a}^{3} \Pi_{\mathrm{u}}$ states are listed in addition. All values are given in $\mathrm{cm}^{-1} . T$ is relative to the $\mathrm{a}^{3} \Pi_{\mathrm{u}}, v=0$ state. Uncertainties are one standard deviation.

\begin{tabular}{lcccc}
\hline \hline Parameter & $1{ }^{5} \Pi_{\mathrm{u}}$ & $1{ }^{5} \Pi_{\mathrm{g}}{ }^{\mathrm{a}}$ & $\mathrm{d}^{3} \Pi_{\mathrm{g}}{ }^{\mathrm{b}}$ & $\mathrm{a}^{3} \Pi_{\mathrm{u}}{ }^{\mathrm{b}}$ \\
\hline$T_{0}$ & $51049.7995(48)$ & $29258.5922(48)$ & $19378.46446(30)$ & 0 \\
$B_{0}$ & $1.21905(21)$ & $1.14413(11)$ & $1.7455695(20)$ & $1.6240451(22)$ \\
$A_{0}$ & $8.1983(37)$ & $8.9450(47)$ & $-14.00111(28)$ & $-15.26912(20)$ \\
$\lambda_{0}$ & $0.0350(15)$ & $-0.0428(23)$ & $0.03303(20)$ & $-0.15490(25)$ \\
$o_{0}$ & $-0.0343(19)$ & $-0.0744(39)$ & $0.61085(22)$ & $0.67539(20)$ \\
$D_{0} \times 10^{6}$ & $10(1)$ & $\ldots$ & $6.82103(66)$ & $6.45068(84)$ \\
\hline \hline
\end{tabular}

${ }^{a}$ Reference 5 .

${ }^{\mathrm{b}}$ Reference 25 .

too large (vide infra). In fact, a variation of $D$ in the range of $5-12 \times 10^{-6} \mathrm{~cm}^{-1}$ had only little effect on the fitted rotational constants and the resulting rms value.

For comparison, the molecular constants for the $1{ }^{5} \Pi_{\mathrm{g}}$, $v=0$ state are listed that have been obtained by the deperturbation study of the $\mathrm{d}^{3} \Pi_{\mathrm{g}}, v=6$ state..$^{5}$ Additionally, the constants for the lower and upper electronic states of the Swan system are given. The valence state configuration of the $1^{5} \Pi_{\mathrm{u}}$ state may be written as $\sigma_{\mathrm{g}}^{2} \sigma_{\mathrm{u}} \pi_{\mathrm{u}}^{2} \sigma_{\mathrm{g}}^{2} \pi_{\mathrm{g}}$ arising from the promotion of an electron $\sigma_{\mathrm{u}} \rightarrow \sigma_{\mathrm{g}}$ from the lower $1{ }^{5} \Pi_{\mathrm{g}}$ configuration, i.e., $\sigma_{\mathrm{g}}^{2} \sigma_{\mathrm{u}}^{2} \pi_{\mathrm{u}}^{2} \sigma_{\mathrm{g}} \pi_{\mathrm{g}}$. Nominally, the same electron promotion is taking place in the Swan system. From the a ${ }^{3} \Pi_{\mathrm{u}}$ electronic valence configuration $\sigma_{\mathrm{g}}^{2} \sigma_{\mathrm{u}}^{2} \pi_{\mathrm{u}}^{3} \sigma_{\mathrm{g}}$, the $\sigma_{\mathrm{u}} \rightarrow \sigma_{\mathrm{g}}$ promotion leads to the $\mathrm{d}^{3} \Pi_{\mathrm{g}}$ configuration $\sigma_{\mathrm{g}}^{2} \sigma_{\mathrm{u}} \pi_{\mathrm{u}}^{3} \sigma_{\mathrm{g}}^{2}$. A comparison of the molecular constants in Table III reflects the similarity of the quintet $\Pi_{\mathrm{u}} \leftarrow \Pi_{\mathrm{g}}$ and triplet $\Pi_{\mathrm{g}} \leftarrow \Pi_{\mathrm{u}}$ transitions. They occur both at an electronic excitation energy of $\approx 20000 \mathrm{~cm}^{-1}$. Furthermore, the rotational constants increase roughly by $7 \%$ from the initial to the excited states for both transitions. Similarly, the spin-orbit constants decrease equally in magnitude by roughly $8 \%$.

\section{Ab initio computations}

$A b$ initio calculations have been performed by following closely the methodology of recent high-level studies of $\mathrm{C}_{2}{ }^{7,28}$ Potential energies were computed between 0.9 and $3.0 \AA$ by $0.05 \AA$ steps at the multi-reference configuration interaction (MRCI) level of theory as implemented in the MOLPRO ${ }^{29}$ suite of programs. To set up the CI expansion, active orbital spaces and reference states were obtained in $\mathrm{D}_{2 \mathrm{~h}}$ symmetry by applying complete active space self-consistent field (CASSCF) theory. ${ }^{30}$ The CASSCF states were generated in the full valence space $((8,8)$-CAS $)$ by equally averaged calculations including the ${ }^{1} \mathrm{~A}_{\mathrm{g}},{ }^{3} \mathrm{~B}_{3 \mathrm{u}}, \mathrm{B}_{3 \mathrm{u}},{ }^{3} \mathrm{~B}_{2 \mathrm{u}},{ }^{5} \mathrm{~B}_{2 \mathrm{u}},{ }^{3} \mathrm{~B}_{2 \mathrm{~g}},{ }^{5} \mathrm{~B}_{2 \mathrm{~g}},{ }^{3} \mathrm{~B}_{3 \mathrm{~g}}$, and ${ }^{5} B_{3 g}$ states. $D_{2 h}$ symmetry labels correlate with $D_{\infty \mathrm{h}}$ labels as follows: $A_{g} \rightarrow \Sigma_{g}^{+}, B_{2 g}+B_{3 g} \rightarrow \Pi_{g}, B_{2 u}+B_{3 u} \rightarrow \Pi_{u}$.

The MRCI states were then obtained from contractions in the space of a given symmetry species. ${ }^{31-34}$ These calculations were performed using the aug-cc-pCV6Z basis set and correlating all electrons (8 valence and 4 core electrons). In addition, the Davidson correction for quadruple excitation is included in the results.

The computed potentials for the a ${ }^{3} \Pi_{\mathrm{u}}, \mathrm{d}^{3} \Pi_{\mathrm{g}}, 1{ }^{5} \Pi_{\mathrm{g}}$, and $1{ }^{5} \Pi_{\mathrm{u}}$ are shown in Fig. 1 and summarized in detail in Table IV.
The equilibrium bond length has been obtained by energy optimization using finite differences as implemented in the MOLPRO $^{29}$ package at the above mentioned level of theory and basis set. The vibrational term energies $T_{0}$ in Table IV are relative to the $v=0$ ground state of the a ${ }^{3} \Pi_{\mathrm{u}}$ state which is computed to be $\approx 818 \mathrm{~cm}^{-1}$ above its potential minimum.

Vibrational-rotational levels were computed by solving the one-dimensional radial Schrödinger equation on each $a b$ initio potential curve by using the LEVEL ${ }^{35}$ computer program. For the numerical integration, the potential energies from the $a b$ initio calculations have been interpolated using cubic splines with a $0.001 \AA$ mesh size. The spectroscopic constants listed in Table IV have been obtained by a nonlinear least-squares fit to the expression, consistent with a $\mathbf{N}^{2}$ Hamiltonian ${ }^{27}$

$$
\begin{aligned}
G(v)= & T_{e}+\omega_{e}\left(v+\frac{1}{2}\right)+\omega_{e} x_{e}\left(v+\frac{1}{2}\right)^{2} \\
& +\omega_{e} y_{e}\left(v+\frac{1}{2}\right)^{3}+\omega_{e} z_{e}\left(v+\frac{1}{2}\right)^{4} \\
& +\left[B_{e}+\alpha\left(v+\frac{1}{2}\right)+\gamma\left(v+\frac{1}{2}\right)^{2}\right. \\
& \left.+\delta\left(v+\frac{1}{2}\right)^{3}\right] J(J+1) \\
& +\left[D_{e}+\beta\left(v+\frac{1}{2}\right)\right] J^{2}(J+1)^{2} .
\end{aligned}
$$

It is important to mention that this expansion is strictly valid only when $J=R=N$, i.e., in the case of a ${ }^{1} \Sigma$-type state. For the ${ }^{3} \Pi$ and ${ }^{5} \Pi$ states discussed in this study, spin-orbit and minor effects such as $\lambda$-doubling coupling terms need to be added to the Hamiltonian. However, the inclusion of spin-orbit matrix elements was found to result in small changes of the vibrational energy levels only. ${ }^{36}$ For the a ${ }^{3} \Pi_{\mathrm{u}}$ and $\mathrm{d}^{3} \Pi_{\mathrm{g}}$ states, energy corrections of less than $0.01 \mathrm{~cm}^{-1}$ have been computed. Taking into account the decreased magnitude of the spin-orbit values, $A$, of the two quintet states relative to the values of the a ${ }^{3} \Pi_{\mathrm{u}}$ and $\mathrm{d}^{3} \Pi_{\mathrm{g}}$ triplet states (Table III), an even smaller effect is expected and, therefore, omitted in the present data reduction for all states.

Considering these limitations, the theoretical constants obtained from the vibrational energy levels and wave functions are in good overall agreement with the experimentally determined constants (Table IV). The accuracy of the MRCI computation is well demonstrated by inspection of the molecular constants of the Swan system. While the calculated potential of the a ${ }^{3} \Pi_{u}$ state yields molecular constants which 
TABLE IV. Computed spectroscopic constants for the $\mathrm{a}^{3} \Pi_{\mathrm{u}}, \mathrm{d}^{3} \Pi_{\mathrm{g}}, 1^{5} \Pi_{\mathrm{g}}$, and $1^{5} \Pi_{\mathrm{u}}$ states.

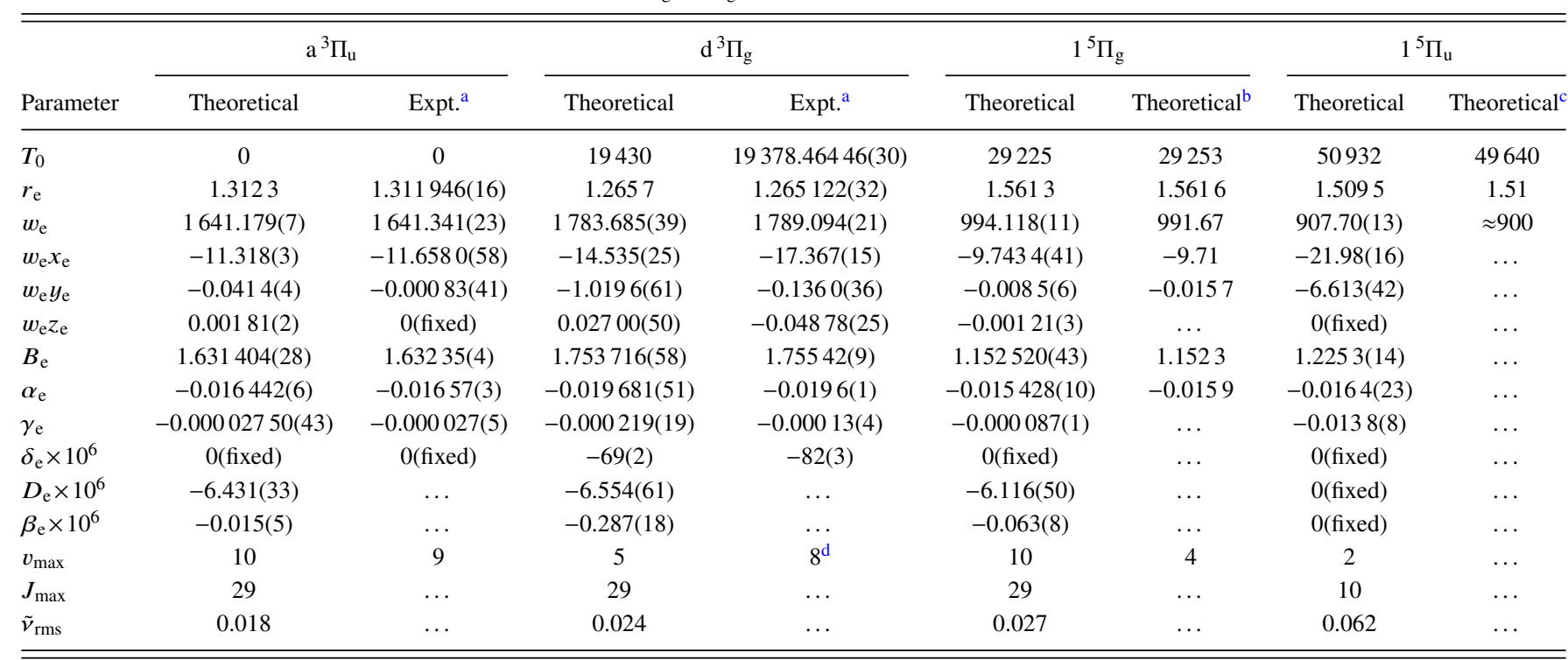

${ }^{\mathrm{a}}$ Reference 25 .

${ }^{\mathrm{b}}$ Reference 7, $a b$ initio, aug-cc-pV6Z + Davidson's correction. Core-valence effect and relativistic corrections with aug-cc-pVQZ.

${ }^{\mathrm{c}}$ Reference 38 .

${ }^{\mathrm{d}} v=1,2,3,5,7,8$.

we consider to agree rather well with the experiment, small discrepancies are present for the $d^{3} \Pi_{g}$ state. One possible reason for the deviations has recently been discussed, ${ }^{28}$ as the $\mathrm{d}^{3} \Pi_{\mathrm{g}}$ state has an avoided crossing with the e ${ }^{3} \Pi_{\mathrm{g}}$ state. ${ }^{37}$ As a consequence, only vibrational levels up to $v_{\max }=5$ are included that are located well below the interaction region of the $\mathrm{d}^{3} \Pi_{\mathrm{g}}$ and $\mathrm{e}^{3} \Pi_{\mathrm{g}}$ states. The resulting fit yields a fairly good agreement with the experimental data. The differences can in principle be reduced further, if data up to $v_{\max }=7$ are considered, and the expansion defined in Eq. (14) is enlarged to accommodate higher vibrational levels at the presence of the avoided crossing. Note, however, that the present adjustment procedure is different from the adjustment carried out in Ref. 25, where molecular constants have been fitted to $v$-dependent parameters used in spin-orbit and $\lambda$-doubling Hamiltonians.

The values of the lower quintet state $1{ }^{5} \Pi_{\mathrm{g}}$ can be compared to the $T_{0}$ value in Table III and the ab initio computation of Schmidt and Bacskay. ${ }^{7}$ These authors have performed MRCI computations with Davidson's correction using the augcc-pV6Z basis set and have included core-valence correlation and relativistic corrections with the quadruple-zeta basis. In this work, core-valence effects are included by applying the same correlation consistent basis set (aug-cc-pCV6Z) in MOLPRO. As listed in Table IV, only minor differences are obtained for the molecular constants.

The comparison between the different theoretical approaches and the experimental findings shows only minor differences in the molecular constants. Table IV validates therefore the accuracy of the applied method for the computation of the excited $1{ }^{5} \Pi_{u}$ state of $C_{2}$. Note, however, that the potential of this state is special as it is predissociative (see Fig. 1). The shallow potential only accommodates three vibrational levels. The barrier is the consequence of an avoided crossing with a higher level of the same symmetry species (henceforth called $2^{5} \Pi_{u}$ ), which has a minimum roughly at $1.9 \AA$ and lies about $18100 h c \mathrm{~cm}^{-1}$ above the top of the barrier of the $1{ }^{5} \Pi_{u}$ state; this energy gap increases to about $32800 \mathrm{hc} \mathrm{cm}-1$ in the asymptotic region of separate atoms (these energies were calculated in a preliminary work with an aug-cc-pVQZ basis and the corresponding potential function is not reproduced in Fig. 1). In addition, a curve crossing with the $1{ }^{5} \Delta_{\mathrm{g}}$ near the equilibrium distance has been suggested in earlier MRCI computations. ${ }^{38}$ Nevertheless, by solving the radial Schrödinger equation to obtain the vibrational levels and their wavefunctions, a fit to Eq. (14) is feasible and yields the spectroscopic constants listed in Table IV. Even though a relatively large rms error has to be considered, the result is strongly indicative of the vibrational quanta observed in the $1{ }^{5} \Pi_{\mathrm{u}}$ state. The $T_{0}$ term value of $50932 \mathrm{~cm}^{-1}$ is in good agreement with the experimentally determined value of $51050 \mathrm{~cm}^{-1}$. For comparison, the $T_{1}$ term value of $51774 \mathrm{~cm}^{-1}$ is $\approx 842 \mathrm{~cm}^{-1}$ higher rendering the $v=1$ vibrational level clearly not a viable candidate for the measured transition. Furthermore, the experimental value of $B_{0} \approx 1.219 \mathrm{~cm}^{-1}$ (Table III) compares well with the theoretical value of the rotational constant for the zero-point energy level of $\approx 1.215 \mathrm{~cm}^{-1}$ which is obtained by solving the radial Schrödinger equation for $v=0$. Again, the corresponding value $B_{1}$ for the $v=1$ vibrational level amounts to $1.171 \mathrm{~cm}^{-1}$. Considering an accuracy of $\approx 0.1 \%$, the value is significantly lower than the experimental value. Thus, on the basis of the term and rotational constant values, it is concluded that the observed level of the $1^{5} \Pi_{u}$ is $v=0$.

As discussed above, the $\sigma_{\mathrm{u}} \rightarrow \sigma_{\mathrm{g}}$ electron promotion upon excitation of the $1{ }^{5} \Pi_{\mathrm{u}} \leftarrow 1{ }^{5} \Pi_{\mathrm{g}}$ transition is comparable to the change of the electronic valence configuration in the Swan band system. While in the latter, the vibrational frequency is increased by $\approx 8 \%$, a decrease of similar magnitude is computed for the $1{ }^{5} \Pi_{u}-1{ }^{5} \Pi_{g}$ transition. By 
considering the Kratzer equation

$$
D_{\mathrm{e}}=\frac{4 B_{\mathrm{e}}^{3}}{\omega_{\mathrm{e}}^{2}},
$$

the weakening of the bond strength upon excitation of the $1{ }^{5} \Pi_{\mathrm{u}}$ is accompanied by an increase of the centrifugal distortion constant. Considering $B_{\mathrm{e}}$ and $\omega_{\mathrm{e}}$ for the $1{ }^{5} \Pi_{\mathrm{u}}$ state in Table IV, a value for $D_{\mathrm{e}} \approx 8.93 \times 10^{-6} \mathrm{~cm}^{-1}$ is evaluated. This value is significantly higher than the corresponding value of the $1^{5} \Pi_{\mathrm{g}}$ state $\left(\approx 6.12 \times 10^{-6} \mathrm{~cm}^{-1}\right)$. In contrast, the Swan levels $\mathrm{d}^{3} \Pi_{\mathrm{g}}$ and $\mathrm{a}^{3} \Pi_{\mathrm{u}}$ exhibit closely matching values for the centrifugal distortion constant. Inspection of the values obtained by solving the radial Schrödinger equation shows accordingly that the $D_{0}$ values increase significantly from 6.21 to $9.58 \times 10^{-6} \mathrm{~cm}^{-1}$ and rationalizes the observed $D_{0}$ of $\approx 10 \times 10^{-6} \mathrm{~cm}^{-1}$ for the $1^{5} \Pi_{\mathrm{u}}$ state (Table III).

\section{SUMMARY AND CONCLUSION}

In this work, we report on the first high-spin transition of $\mathrm{C}_{2}$. Its detection constitutes a rare exception in such a simple system exhibiting a ${ }^{1} \Sigma_{\mathrm{g}}^{+}$ground state. To the best of our knowledge, only one comparable quintet-quintet transition has so far been reported in the literature, the so-called Herman infrared (HIR) band of $\mathrm{N}_{2}$ at $\approx 12000 \mathrm{~cm}^{-1}$, a $1{ }^{5} \Pi_{\mathrm{u}}-1^{5} \Sigma_{\mathrm{g}}^{+}$ transition. In fact, most low-lying valence quintet states are repulsive with the exception of the $1^{5} \Pi_{\mathrm{g}}$ and the van der Waals bond of $1{ }^{5} \Sigma^{+}$of CO as well as the weakly bound $1^{5} \Pi_{\mathrm{g}}$ state in $\mathrm{O}_{2}\left(D_{\mathrm{e}} \approx 0.16 \mathrm{eV}\right)$ (Ref. 39 and references therein).

The observation of the $1{ }^{5} \Pi_{\mathrm{u}}-1{ }^{5} \Pi_{\mathrm{g}}$ transition has been carried out by PFOODR spectroscopy by applying non-linear TC-RFWM. Strongly mixed "gateway" states exhibiting both triplet and quintet character are excited from the thermally populated a ${ }^{3} \Pi_{\mathrm{u}}, v=5$ state. From these intermediate levels, 57 rotational transitions are observed that reveal a clear pattern of the five sub-bands constituting a ${ }^{5} \Pi_{\mathrm{u}}-{ }^{5} \Pi_{\mathrm{g}}$ transition. Accurate molecular constants for the upper state $\left(1^{5} \Pi_{u}\right)$ are determined by a least-squares optimization procedure to a model of a conventional Hamiltonian for a linear molecule.

Figure 4 depicts the position of the high-spin transition of the dicarbon molecule relative to the known singlet and triplet manifolds.

The experimental results are compared by performing high-level $a b$ initio calculations at the MRCI level of theory using a large correlation consistent basis set (aug-cc-pCV6Z). The accuracy of the method is assessed by applying the method to the Swan levels a ${ }^{3} \Pi_{u}$ and $d^{3} \Pi_{g}$ and comparison of the results with experimentally determined molecular constants. In addition, good agreement with earlier calculations is found for the computation of the lower quintet state $\left(1^{5} \Pi_{\mathrm{g}}\right)$. The theoretical potential of the $1{ }^{5} \Pi_{u}$ is found to be predissociative and displays a shallow potential accommodating three vibrational levels only.

Presently, the ab initio computations are repeated by using the MRCI-F12 method and applying the largest possible basis currently implemented in the 2012 version of MOLPRO. ${ }^{29}$ The quality of this calculation is equal or superior to that with the pCV6Z basis set. For instance, the $T_{0}$ value of the

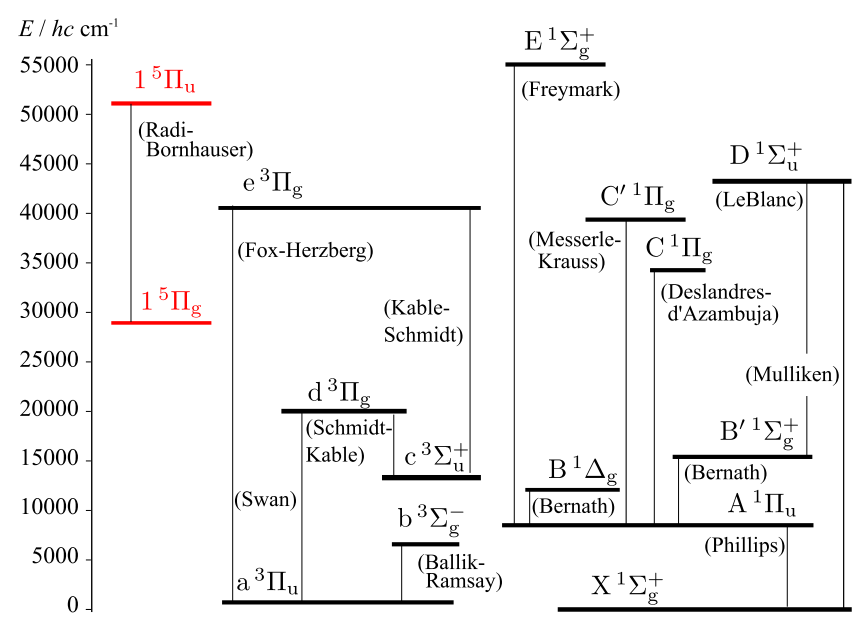

FIG. 4. Low lying singlet, triplet, and quintet electronic states of $\mathrm{C}_{2}$.

$1{ }^{5} \Pi_{\mathrm{g}}$ and $1{ }^{5} \Pi_{\mathrm{u}}$ levels is 29304 and $51012 \mathrm{~cm}^{-1}$, respectively, from preliminary calculations. The implicit correlation of the electrons significantly reduces computation times. The results will be published in a forthcoming publication focusing on the vibrational analysis of the $1{ }^{5} \Pi_{\mathrm{u}}-1{ }^{5} \Pi_{\mathrm{g}}$ transition.

It is interesting to mention that the characterization of the newly found $1{ }^{5} \Pi_{\mathrm{u}}$ opens ways to observe further transitions in the quintet manifold. In particular, the $1^{5} \Pi_{\mathrm{u}}-1{ }^{5} \Sigma_{\mathrm{g}}^{+}$transition should be observable at $\approx 11500 \mathrm{~cm}^{-1},{ }^{38}$ which is the analog to the HIR band of $\mathrm{N}_{2}$.

\section{ACKNOWLEDGMENTS}

This work is supported by the Swiss National Science Foundation (\#200020_146387 and \#200021_153170) and from CNRS, Région Alsace and the French Ministry for Research. We also thank Dr. Chantal Daniel for stimulating remarks and help.

${ }^{1}$ W. H. Wollaston, Philos. Trans. R. Soc. London 92, 365 (1802).

${ }^{2}$ W. Swan, Ann. Phys. 176, 306 (1857).

${ }^{3}$ M. Martin, J. Photochem. Photobiol., A 66, 263 (1992).

${ }^{4}$ D. L. Kokkin, N. J. Reilly, C. W. Morris, M. Nakajima, K. Nauta, S. H. Kable, and T. W. Schmidt, J. Chem. Phys. 125, 231101 (2006).

${ }^{5}$ P. Bornhauser, Y. Sych, G. Knopp, T. Gerber, and P. Radi, J. Chem. Phys. 134, 044302 (2011).

${ }^{6}$ A. Fowler, Mon. Not. R. Astron. Soc. 70, 484 (1910).

${ }^{7}$ T. W. Schmidt and G. B. Bacskay, J. Chem. Phys. 134, 224311 (2011).

${ }^{8}$ K. Kirby and B. Liu, J. Chem. Phys. 70, 893 (1979).

${ }^{9}$ C. E. Little and P. G. Browne, Chem. Phys. Lett. 134, 560 (1987).

${ }^{10}$ P. Bornhauser, G. Knopp, T. Gerber, and P. Radi, J. Mol. Spectrosc. 262, 69 (2010).

${ }^{11}$ L. Li and R. W. Field, J. Phys. Chem. 87, 3020 (1983).

${ }^{12}$ X. Xie and R. Field, J. Mol. Spectrosc. 117, 228 (1986).

${ }^{13}$ H.-L. Dai and R. W. Field, Molecular Dynamics and Spectroscopy by Stimulated Emission Pumping (World Scientific, 1995).

${ }^{14}$ F. Xie, D. Li, L. Li, R. W. Field, and S. Magnier, Chem. Phys. Lett. 431, 267 (2006).

${ }^{15}$ J. Magnes, E. Ahmed, C. Goldberg, A. M. Lyyra, S. Magnier, M. AubertFrécon, Y. Liu, and L. Li, J. Mol. Spectrosc. 221, 72 (2003).

${ }^{16}$ G. Jong, L. Li, T. J. Whang, W. C. Stwalley, J. A. Coxon, M. Li, and A. M. Lyyra, J. Mol. Spectrosc. 155, 115 (1992).

${ }^{17}$ N. Bartels, T. Schäfer, J. Hühnert, R. W. Field, and A. M. Wodtke, J. Chem. Phys. 136, 214201 (2012).

${ }^{18}$ Y. Sych, P. Bornhauser, G. Knopp, Y. Liu, T. Gerber, R. Marquardt, and P. P. Radi, J. Chem. Phys. 139, 154203 (2013). 
${ }^{19}$ M. Tulej, M. Meisinger, G. Knopp, A. M. Walser, T. Gerber, and P. P. Radi, J. Raman Spectrosc. 38, 1022 (2007).

${ }^{20}$ M. Tulej, G. Knopp, T. Gerber, and P. P. Radi, J. Raman Spectrosc. 41, 853 (2010).

${ }^{21}$ H. Linnartz, Phys. Scr. 69, C37 (2004).

${ }^{22}$ T. Muller and P. H. Vaccaro, Chem. Phys. Lett. 266, 575 (1997).

${ }^{23}$ P. P. Radi, H. M. Frey, B. Mischler, A. P. Tzannis, P. Beaud, and T. Gerber, Chem. Phys. Lett. 265, 271 (1997).

${ }^{24}$ S. Williams, J. D. Tobiason, J. R. Dunlop, and E. A. Rohlfing, J. Chem. Phys. 102, 8342 (1995)

${ }^{25}$ A. Tanabashi, T. Hirao, T. Amano, and P. F. Bernath, Astrophys. J., Suppl. Ser. 169, 472 (2007)

${ }^{26} \mathrm{C}$. M. Western, PGOPHER, a program for simulating rotational structure, University of Bristol, 2012

${ }^{27}$ J. M. Brown and A. J. Merer, J. Mol. Spectrosc. 74, 488 (1979).

${ }^{28}$ M. Nakajima and Y. Endo, J. Chem. Phys. 139, 244310 (2013).
${ }^{29}$ H.-J. Werner, P. J. Knowles, G. Knizia, F. R. Manby, M. Schütz et al., MOLPRo, version 2010.1, a package of ab initio programs, 2010, see http://www. molpro.net.

${ }^{30}$ H.-J. Werner and W. Meyer, J. Chem. Phys. 74, 5794 (1981).

${ }^{31}$ H.-J. Werner and P. J. Knowles, J. Chem. Phys. 82, 5053 (1985).

${ }^{32}$ P. J. Knowles and H.-J. Werner, Chem. Phys. Lett. 145, 514 (1988).

${ }^{33}$ H.-J. Werner and P. J. Knowles, J. Chem. Phys. 89, 5803 (1988).

${ }^{34}$ H.-J. Werner and P. J. Knowles, Theor. Chim. Acta 78, 175 (1990).

${ }^{35}$ R. Le Roy, University of Waterloo Chemical Physics Research Report CP663, 2007.

${ }^{36}$ D. L. Kokkin, G. B. Bacskay, and T. W. Schmidt, J. Chem. Phys. 126, 084302 (2007)

${ }^{37}$ M. Nakajima, J. A. Joester, N. I. Page, N. J. Reilly, G. B. Bacskay, T. W. Schmidt, and S. H. Kable, J. Chem. Phys. 131, 044301 (2009).

${ }^{38}$ P. Bruna and F. Grein, Can. J. Phys. 79, 653 (2001).

${ }^{39}$ P. J. Bruna and F. Grein, Can. J. Phys. 87, 589 (2009). 